\section{Crisis neuropática por suspensión de nitisinona en una paciente con tirosinemia: informe de un caso}

Ibarra-González $\mathrm{I}^{1}$, Belmont-Martínez $\mathrm{L}^{2}$, Cervantes-Bustamante $\mathrm{R}^{3}$, ZárateMondragón $\mathrm{F}^{3}$, Guillén-López $\mathrm{S}^{2}$, Fernández-Lainez $\mathrm{C}^{2}$, Revilla-Estivil $\mathrm{N}^{4}$, Vela- Amieva $\mathrm{M}^{2}$

\section{Resumen}

Se presenta el caso de una paciente con tirosinemia hepatorrenal (TYR1) que, debido a la interrupción por cuatro semanas de tratamiento con nitisinona, tuvo una grave crisis neurológica de pseudoporfiria caracterizada por vómito, dolor abdominal, irritabilidad e hipertensión arterial, con gran elevación de la succinilacetona y alfafetoproteína. La crisis requirió tratamiento intrahospitalario. Una semana después de reiniciar la administración del fármaco revirtió totalmente. Este caso enfatiza la importancia de mantener el tratamiento ininterrumpido con nitisinona en pacientes con tirosinemia hepatorrenal. También es útil para reconocer el cuadro clínico y la fisiopatología de las crisis neurológicas características de esta enfermedad.

PALABRAS CLAVE: tirosinemia hepatorrenal, nitisinona, succinilacetona, crisis neurológica.

Acta Pediatr Mex. 2017 Sep;38(5):322-329.

\section{Neurologic crises after interruption of nitisinone in a tyrosinemia patient: case report.}

Ibarra-González I', Belmont-Martínez $\mathrm{L}^{2}$, Cervantes-Bustamante $\mathrm{R}^{3}$, ZárateMondragón $\mathrm{F}^{3}$, Guillén-López $\mathrm{S}^{2}$, Fernández-Lainez $\mathrm{C}^{2}$, Revilla-Estivil $\mathrm{N}^{4}$, Vela- Amieva $\mathrm{M}^{2}$

\begin{abstract}
We present the case of a female patient with hepatorenal tyrosinemia who presented a severe porphyria-like neurologic crisis four weeks after interrupting nitisinone treatment. The crisis was characterized by vomiting, abdominal pain, irritability, and high blood pressure, with elevation of blood succinylacetone and alpha-fetoprotein. The crisis required hospitalization and reverted totally one week after the reintroduction of nitisinone. This clinical case emphasizes the importance of maintaining nitisinone treatment uninterruptedly. This paper is useful for recognizing the clinical picture of the neurologic crises of TYR-1 and its physiopathology.
\end{abstract}

KEYWORDS: hepatorenal tyrosinemia; nitisinone; NTBC; succinylacetone; neurologic crises
${ }^{1}$ Unidad de genética de la Nutrición, Instituto de Investigaciones Biomédicas, UNAM.

${ }^{2}$ Laboratorio de Errores Innatos del Metabolismo y Tamiz, Instituto Nacional de Pediatría.

${ }^{3}$ Servicio de Gastronutrición, Instituto Nacional de Pediatría.

${ }^{4}$ Departamento de urgencias, Instituto Nacional de Pediatría.

Recibido: 6 de junio del 2016

Aceptado: 5 de diciembre del 2016

Correspondencia

Dra. Marcela Vela-Amieva.

dravelaamieva@yahoo.com

Este artículo debe citarse como Ibarra-González I, Belmont-Martínez L, CervantesBustamante R, Zárate-Mondragón F, Guillén-López $S$, Fernández-Lainez $C$ et al. Crisis neuropática por suspensión de nitisinona en una paciente con tirosinemia: informe de un caso. Acta Pediatr Mex. 2017;38(5):322-329. 


\section{INTRODUCCIÓN}

La tirosinemia tipo 1 o tirosinemia hepatorrenal (TYR-1) (OMIM 276700) es un error innato del metabolismo de los aminoácidos ocasionado por deficiencia de la fumarilacetoacetato hidrolasa, última enzima del ciclo catabólico de la tirosina (Figura 1). Su prevalencia global es de uno en 100,000 a 120,000 recién nacidos vivos, ${ }^{1,2}$ aunque existen áreas geográficas como
Quebec, Canadá, donde la frecuencia es mucho mayor, uno en 1846 recién nacidos vivos. ${ }^{3,4}$ En México, la prevalencia se desconoce por la falta de tamiz neonatal obligatorio para tirosinemia hepatorrenal, por lo que no existe un registro nacional de casos.

El cuadro clínico se caracteriza por falla hepática con coagulopatía pronunciada acompañada de ascitis y edema, así como signos de raquitismo

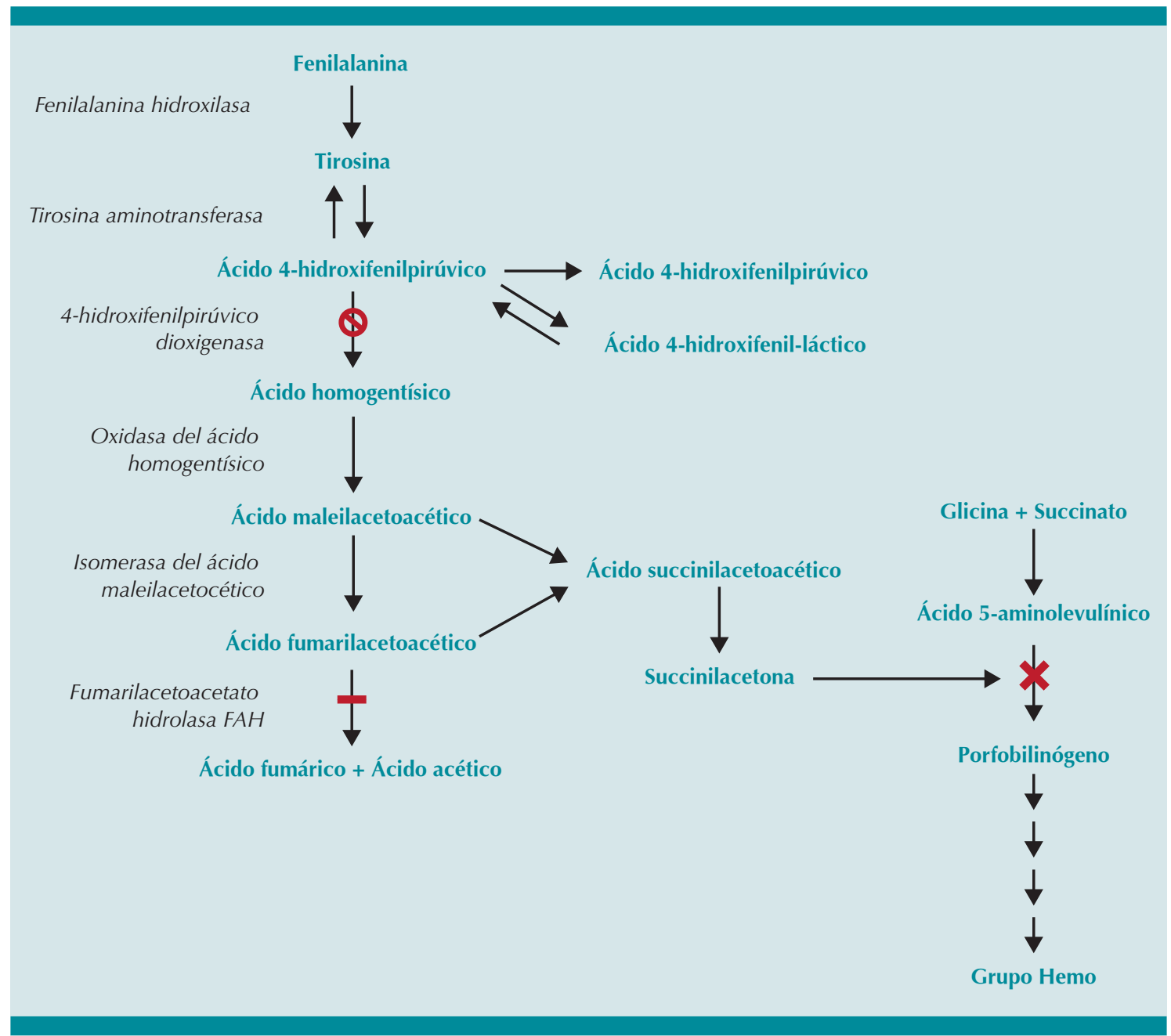

Figura 1. Ruta metabólica de la tirosina. La barra negra señala el sitio del bloqueo de la enzima fumarilacetoacetato hidrolasa, responsable de la tirosinemia hepatorrenal; la cruz muestra el sitio de inhibición del metabolismo del grupo Hemo por acumulación de succinilacetona. El símbolo $\theta$ señala el sitio de acción de la nitisinona. 
hipofosfatémico secundarios a tubulopatía renal con síndrome de Fanconi. También existe transaminasemia e hipoalbuminemia y los pacientes pueden o no tener ictericia, glucosuria, aminoaciduria y fosfaturia. ${ }^{4} \mathrm{~A}$ mediano y largo plazos, pueden ocurrir nefrocalcinosis, gloméruloesclerosis, falla renal crónica, afección de nervios periféricos, cirrosis, nódulos hepáticos y carcinoma hepatocelular. ${ }^{5,6}$ La tirosinemia hepatorrenal se clasifica según la edad de presentación de síntomas: aguda, cuando los síntomas se presentan en los seis primeros meses de vida; subaguda, que se inicia entre los seis y los 12 meses de edad y crónica, después del año de vida. Mientras más temprano comienzan los síntomas, más severa es la enfermedad.

Es bien sabido que cuando estos pacientes no se diagnostican en el periodo neonatal y no reciben tratamiento temprano, tienen una alta mortalidad..$^{7-9}$ En México se ha descrito la elevada mortalidad de la tirosinemia hepatorrenal..$^{10}$ La succinilacetona es el metabolito patognomónico de la tirosinemia hepatorrenal ${ }^{11}$ y el patrón bioquímico de la enfermedad incluye hipertirosinemia, hipermetioninemia, acumulación de los ácidos fumarilacetoacético y maleilacetoacético en la sangre y en orina, los cuales juegan un papel crucial en el daño hepático, renal y neurológico de los pacientes. Como indicador, la elevación aislada de tirosina carece de sensibilidad, ya que puede deberse a un gran número de patologías o condiciones médicas como el ayuno, uso de nutrición parenteral total, tirosinemia transitoria del neonato y hepatopatías en general, de causa congénita o adquirida. ${ }^{1,9} \mathrm{Es}$ muy importante considerar que la alfa-fetoproteína, que es un biomarcador tumoral, suele tener niveles promedio de $160,000 \mathrm{ng} / \mathrm{mL}$ en etapas muy tempranas del padecimento. ${ }^{1,4-8}$

Una de las complicaciones más graves de la tirosinemia hepatorrenal es la presencia de crisis neurológicas o pseudoporfiria ("porphyria-like").
Aunque algunos autores las han descrito desde hace cerca de 50 años, ${ }^{12,13}$ la primera descripción clínica y bioquímica detallada fue realizada por Mitchell y sus colaboradores en $1990 .{ }^{14}$ La sintomatología principal de estas crisis es el dolor en las piernas o abdominal, usualmente acompañado de vómito e íleo paralítico. Existe hipertonía típicamente axial, que varía en severidad, desde una leve resistencia a la flexión del cuello hasta una postura de opistótonos. También ocurre una neuropatía motora aguda ascendente, que puede progresar a parálisis con dificultad respiratoria que requiere ventilación mecánica. ${ }^{6}$ En algunos casos ocurren episodios de automutilación caracterizadas por mordeduras y laceración de la lengua o bruxismo severo que causa avulsión de los dientes. También hay hipertensión arterial y taquicardia.

Los principales datos de laboratorio durante las crisis neurológicas de la tirosinemia hepatorrenal son hiponatremia, hipokalemia, aumento de enzimas hepáticas, tiempo de protrombina prolongado y niveles elevados de ácido delta-aminolevulínico. Ocasionalmente hay hiperamonemia y bicarbonato sérico bajo. ${ }^{14}$

Bioquímicamente, las crisis neurológicas en esta enfermedad se deben a que la acumulación de succinilacetona inhibe la síntesis de porfirina a nivel de la enzima deshidratasa del ácido aminolevulínico y causa acumulación de ácido 5-aminolevulínico (Figura 1), que es un potente neurotóxico responsable de la sintomatología similar a la de la porfiria aguda. ${ }^{1}$

La nitisinona o NTBC (2-(2-nitro4-trifluorometilbenzoil)-1,3-ciclohexanediona) $\left(\right.$ Orfadin $^{\circledR}$ ) es un potente inhibidor de la enzima 4-hidroxifenilpiruvato dioxigenasa (4-HPPD), evitando la degradación de la tirosina y la producción de succinilacetona (Figura 1); desde 1992 se convirtió en el tratamiento de elección de la tirosinemia hepatorrenal, utilizada de ma- 
nera conjunta con la dieta restringida en tirosina. Esta combinación ha reducido la necesidad de trasplante hepático o hepatorrenal y ha mejorado notablemente las tasas de supervivencia de los pacientes. ${ }^{11,15,16}$ Se sabe que tan sólo 24 horas después de la primera dosis de nitisinona la succinilacetona no debe ser detectada en la orina, ni en la sangre; debe encontrarse por debajo del valor de referencia; la coagulopatía suele revertir en 48 horas y los tiempos de coagulación se normalizan en una semana. La disminución de alfa-fetoproteína suele ser logarítmica y tiende a normalizarse después del primer año de tratamiento continuo. ${ }^{4}$ La dosis de nitisinona debe ser individualizada según el peso y el cuadro clínico.

El tratamiento de la tirosinemia hepatorrenal requiere de un plan de nutrición individualizado con modificaciones de acuerdo a la ganancia pondo-estatural del paciente, conforme a las concentraciones de aminoácidos en sangre, particularmente las de tirosina y fenilalanina, siguiendo las recomendaciones de Acosta $y$ sus colaboradores. ${ }^{17}$ Las evaluaciones nutricias se realizan con el parámetro de talla para la edad, y dado que el peso en los pacientes con tirosinemia hepatorrenal puede estar afectado por el edema. La hepatomegalia y la ascitis, el perímetro del brazo y el pliegue cutáneo tricipital se toman como marcadores de repleción nutricional.

\section{CASO CLÍNICO}

Niña de cuatro años de edad, producto de un embarazo normoevolutivo no planeado, de padres no consanguíneos aparentemente sanos. Tuvo el antecedente de dolor abdominal e íleo a los ocho meses de edad, que requirió hospitalización por ocho días, manejado como probable oclusión intestinal y que se resolvió con ayuno. A la edad de 9 meses los padres notaron aumento del perímetro abdominal y estreñimiento, por lo que acudieron a un hospital general en donde diagnosticaron intolerancia a la lactosa, que fue tratada con laxantes y fórmula libre de lactosa, sin respuesta favorable. Un ultrasonido abdominal reveló hepatomegalia, por lo cual fue enviada a un servicio de pediatría donde se hallaron pruebas de función hepática anormales, plaquetopenia y tiempos de coagulación prolongados que requirieron transfusión de tres unidades de plasma, con el diagnóstico de hepatitis de etiología a determinar. Una biopsia hepática fue sugestiva de tirosinemia hepatorrenal; sin embargo, no recibió tratamiento específico.

A los dos años de vida fue enviada a nuestra institución, donde se corroboró el diagnóstico de tirosinemia hepatorrenal por la presencia de succinilacetona de $3.6 \mu \mathrm{M}$ (V.R. $<1.0 \mu \mathrm{M})$, tirosina $342 \mu \mathrm{M}$ (V.R. < $169 \mu \mathrm{M})$ en sangre, así como alfafetoproteína de 45,364 Ul/mL (V.R. 0.5-5.5 Ul/mL). Se le trató con dieta restringida en tirosina y alimento libre de fenilalanina y tirosina (Tyrex-2) (Cuadro 1), así como nitisinona: $1 \mathrm{mg} / \mathrm{kg} /$ día, que inicialmente recibió por 11 meses. Sin embargo, como dicho medicamento pertenece a la categoría de fármacos huérfanos, su adquisición por nuestra institución fue compleja y la paciente se quedó sin tratamiento por un mes.

Al principio de la quinta semana de haber suspendido la nitisinona acudió al servicio de urgencias del Instituto Nacional de Pediatría por vómito postprandial y diarrea de dos días acompañada de fiebre de $38^{\circ} \mathrm{C}$ en una sola ocasión; dolor abdominal, astenia, adinamia, insomnio e irritabilidad, tos productiva con rinorrea hialina y tiros intercostales. En la exploración física presentó taquipnea, e hipertensión (142/72 mmHg), hepatomegalia de $2 \mathrm{~cm}$ por debajo del reborde costal. La hipertensión fue persistente y llegó hasta $130 / 90$ y $142 / 72 \mathrm{mmHg}$, lo que requirió tratamiento con prazosina $250 \mathrm{mcg}$ cada $6 \mathrm{~h}$, enalapril, $5 \mathrm{mg}$ cada $24 \mathrm{~h}$, furosemide, $10 \mathrm{mg}$ 
Cuadro 1. Planes de alimentación utilizados en el presente caso A): inicial, B): seguimiento

\begin{tabular}{|c|c|c|c|c|c|}
\hline Alimento & Cantidad & PHE/mg & TYR/mg & Proteína/g & Energía/kcal \\
\hline $\begin{array}{l}\text { Fórmula sin fenilalanina } \\
\text { ni tirosina }\end{array}$ & $150 \mathrm{~g}$ & -- & -- & 22.5 & 720 \\
\hline Agua & $600 \mathrm{~mL}(20 \mathrm{oz})$ & \multicolumn{2}{|c|}{--} & -- & -- \\
\hline \multicolumn{2}{|c|}{ Total } & \multicolumn{2}{|c|}{--} & 22.5 & 720 \\
\hline \multicolumn{2}{|c|}{ Recomendaciones 1-4 años ${ }^{17}$} & \multicolumn{2}{|c|}{$450-1050$} & $>30$ & $900-1800$ \\
\hline \multicolumn{6}{|c|}{$\begin{array}{l}\text { B) Dieta de seguimiento utilizada, de } 1700 \mathrm{kcal} \text {, con una osmolaridad de } 732 \mathrm{mOsm} / \mathrm{L} \text {, para la paciente con } \\
4 \text { años cumplidos, relación calórico proteica de } 47: 1 ; 101 \mathrm{kcal} / \mathrm{kg} / \mathrm{d} \text {; proteína: } 2.1 \mathrm{~g} / \mathrm{kg} / \mathrm{d}\end{array}$} \\
\hline Alimento & Cantidad & PHE/mg & TYR/mg & Proteína/g & Energía/kcal \\
\hline $\begin{array}{l}\text { Fórmula sin fenilalanina } \\
\text { ni tirosina }\end{array}$ & $160 \mathrm{~g}$ & -- & -- & 24 & 768 \\
\hline Fórmula de inicio & $21.5 \mathrm{~g}$ (5 medidas) & \multicolumn{2}{|c|}{169} & 2.04 & 112.45 \\
\hline Maicena & $30 \mathrm{~g}$ & \multicolumn{2}{|c|}{--} & -- & 105 \\
\hline Agua & $700 \mathrm{~mL}(23.3 \mathrm{oz}$ ) & \multicolumn{2}{|c|}{--} & -- & -- \\
\hline Verdura & $5 \mathrm{Eq}$ & \multicolumn{2}{|c|}{125} & 2.5 & 50 \\
\hline Fruta & $5 \mathrm{Eq}$ & \multicolumn{2}{|c|}{125} & 2.5 & 300 \\
\hline Cereal & $8 \mathrm{Eq}$ & \multicolumn{2}{|c|}{400} & 4.8 & 240 \\
\hline Grasas & $1 \mathrm{Eq}$ & \multicolumn{2}{|c|}{9} & 0.1 & 60 \\
\hline Alimentos libres $\mathrm{A}^{17}$ & $1 \mathrm{Eq}$ & \multicolumn{2}{|c|}{9} & 0.1 & 65 \\
\hline \multicolumn{2}{|c|}{ Total } & \multicolumn{2}{|c|}{837} & 36.04 & 1700.45 \\
\hline \multicolumn{2}{|c|}{ Recomendaciones 1-4 años ${ }^{17}$} & \multicolumn{2}{|c|}{$450-1050$} & $>30$ & $900-1800$ \\
\hline
\end{tabular}

cada 12 h. No hubo respuesta, por lo que se agregó propranolol $10 \mathrm{mg}$ cada $8 \mathrm{~h}$, losartán 25 mg cada 12 h y amlodipino 5 mg cada 24 h, así como dieta libre en tirosina y fenilalanina.

Los principales resultados de laboratorio se muestran en el Cuadro 2, destacan la hiponatremia y la hipokalemia, así como leve aumento de las enzimas hepáticas, del amonio y de los tiempos de coagulación. El vómito fue persistente y el dolor abdominal requirió opioide (buprenorfina a dosis baja en infusión 4 mcg/ $\mathrm{kg} /$ día) y clonixinato de lisina $1 \mathrm{mg} / \mathrm{kg} /$ dosis IV cada 8 h. Un ecocardiograma mostró hipertrofia ventricular izquierda con función ventricular normal. A los 17 días de internamiento se logró conseguir la nitisinona, que se reinició a $1 \mathrm{mg} /$ kg/día y a las 24 horas después, hubo una notable mejoría clínica, con normalización gradual de la tensión arterial, de los electrolitos séricos y de la succinilacetona (Figura 2). Egresó una semana después del reinicio del medicamento, clínicamente estable.

\section{DISCUSIÓN}

Antes del advenimiento de la nitisinona las crisis neurológicas de pseudoporfiria de la tirosinemia hepatorrenal ocurrían en $42 \%$ de los pacientes y tenían una elevada mortalidad. ${ }^{14}$ En los pacientes que reciben nitisinona de forma continua se evitan dichas crisis. ${ }^{18,19}$ En la literatura existen 
Cuadro 2. Principales resultados de laboratorio observados al momento del ingreso

\begin{tabular}{lccc|}
\hline Analito & Valor observado & Valor de referencia \\
\hline Sodio & 117 & $138-145 \mathrm{mmol} / \mathrm{L}$ \\
\hline Potasio & 2.4 & $3.7-5.0 \mathrm{mmol} / \mathrm{L}$ \\
\hline Cloro & 107 & $96-109 \mathrm{mmol} / \mathrm{L}$ \\
\hline $\begin{array}{l}\text { Aspartato amino } \\
\text { transferasa }\end{array}$ & 47 & $20-60 \mathrm{UI} / \mathrm{L}$ \\
\hline $\begin{array}{l}\text { Alanina amino } \\
\text { transaminasa }\end{array}$ & 58 & $5-45 \mathrm{UI} / \mathrm{L}$ \\
\hline $\begin{array}{l}\text { Gama-glutamil } \\
\text { transferasa }\end{array}$ & 41 & $6-19 \mathrm{UI} / \mathrm{L}$ \\
\hline $\begin{array}{l}\text { Nitrógeno } \\
\text { ureico }\end{array}$ & 11.2 & $5-17 \mathrm{mg} / \mathrm{dL}$ \\
\hline $\begin{array}{l}\text { Creatinina } \\
\text { Glucosa }\end{array}$ & 0.47 & $0.2-0.7 \mathrm{mg} / \mathrm{dL}$ \\
\hline $\begin{array}{l}\text { Alfa fetoproteína } \\
\text { Amonio }\end{array}$ & 63 & $57-115 \mathrm{mg} / \mathrm{dL}$ \\
\hline $\begin{array}{l}\text { Tiempo de } \\
\text { protrombina }\end{array}$ & 50 & $0.5-5.5 \mathrm{UI} / \mathrm{mL}$ \\
\hline $\begin{array}{l}\text { Porcentaje de } \\
\text { tiempo de } \\
\text { protrombina }\end{array}$ & 74.6 & $11-35 \mathrm{~mol} / \mathrm{L}$ \\
\hline
\end{tabular}

dos trabajos recientes que señalan la presencia de crisis de pseudoporfiria por suspensión de nitisinona, una en un paciente africano de 8 meses de edad y otro turco, ambos habían sido oportunamente diagnosticados con tirosinemia hepatorrenal y tratados desde el periodo neonatal. En estos casos, la interrupción del medicamento se debió a negligencia de los padres. ${ }^{20,21}$ Llama la atención que, tanto en nuestro caso como en los descritos en la literatura, los síntomas neurológicos se iniciaron entre la $4^{a} y$ $5^{a}$ semanas después de la interrupción del medicamento; se caracterizaron por dolor abdominal, astenia, adinamia, alteraciones del estado de conciencia, hipertensión arterial acompañados de hiponatremia, hipokalemia, aumento de las enzimas hepáticas y de la succinilacetona. En nuestro paciente, la suspensión del medicamento se debió a la dificultad para adquirir fármacos de alto costo catalogados como huérfanos. En nuestra institución, gracias a la estrategia del Seguro Popular, ${ }^{22}$ la adquisición de este fármaco ya se ha solucionado.

En concordancia con lo señalado por otros autores, ${ }^{20,21}$ la resolución de la crisis con la reintroducción de la nitisinona es rápida; los pacientes recobran parámetros bioquímicos (Figura 2) y clínicos normales en menos de una semana. Es importante mencionar que durante el tiempo que esta paciente suspendió el medicamento, mantuvo la dieta libre de tirosina y fenilalanina; sin embargo, se sabe que esto no es suficiente para reducir los niveles de succinilacetona, puesto que deja de ocurrir el efecto inhibidor de la nitisinona sobre la 4-hidroxifenilpirúvico dioxigenasa. ${ }^{17}$ Otro aspecto a considerar con la suspensión de nitisinona es el aumento del riesgo de desarrollar carcinoma hepatocelular, que es una de las complicaciones más graves de la tirosinemia hepatorrenal.

Finalmente, aunque en nuestro caso el diagnóstico de tirosinemia hepatorrenal ya estaba establecido antes de que aparecieran las crisis

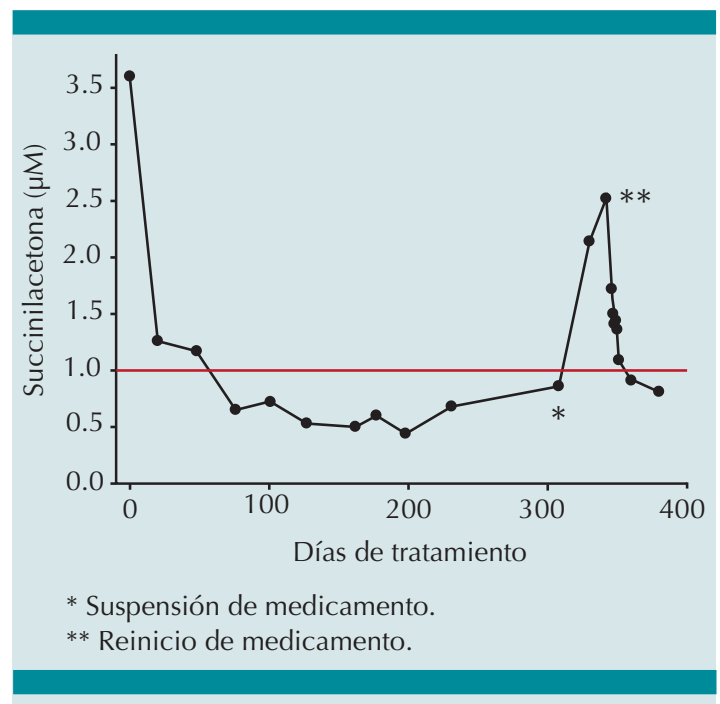

Figura 2. Concentración de succinilacetona en sangre de acuerdo a los días de tratamiento con nitisinona. 
tipo porfiria, es importante destacar que en todo paciente pediátrico con dolor abdominal y manifestaciones neurológicas debe hacerse diagnóstico diferencial con otras patologías tales como síndrome de Guillain-Barré, afección por virus neurotrópicos (polio, coxackie, ecovirus, enterovirus, entre otros), así como neurotoxicidad por plomo, arsénico o talio.

\section{CONCLUSIONES}

El tratamiento con nitisinona debe ser continuo y no debe suspenderse nunca de manera abrupta, pues la suspensión causa elevación de la succinilacetona y otros metabolitos tóxicos, lo que aumenta el riesgo de padecer crisis neurológicas de pseudoporfiria, las cuales tienen elevada mortalidad; dichas crisis revierten en general una semana después de reiniciar el medicamento.

\section{REFERENCIAS}

1. Mitchell GA, Grompe M, Lambert M, Tanguay RM. Hypertyrosinemia. In: Scriver CR, Beaudet AL, Sly WS, Valle D, eds. The Metabolic and Molecular Bases of Inherited Disease. New York, NY; McGraw Hill; 2001:1777-806.

2. Raimann E, Cornejo V, Arias C, Cabello JF, Castro G, Fernández E, et al. Evolución clínica de pacientes chilenos con tirosinemia tipo I tratados con 2-(2-nitro4-trifluorometilbenzoil)1,3-ciclohexanediona (NTBC) Rev Med Chil. 2012;140:169-75.

3. De Braekeleer M, Larochelle J. Genetic Epidemiology of Hereditary Tyrosinemia in Quebec and in SaguenayLac-St-Jean.Am J Hum Genet. 1990;47:302-307.

4. de Laet C, Dionisi-Vici C, Leonard JV, McKiernan P, Mitchell G, Monti L, et al. Recommendations for the management of tyrosinaemia type 1.Orphanet J Rare Dis. doi:10.1186/1750-1172-8-8.

5. El-Karaksy H, Rashed M, El-Sayed R, El-Raziky M, El-Koofy N, El-Hawary $M$, et al. NTBC therapy for tyrosinemia type 1: how much is enough? Eur J Pediatr. 2010;169:689-93.

6. van Spronsen FJ, Thomasse Y, Smit GP, Leonard JV, Clayton PT, Fidler V, et al. Hereditary tyrosinemia type I: a new clinical classification with difference in prognosis on dietary treatment. Hepatology.1994;20:1187-1191.

7. Couce ML, Dalmau J, del Toro M, Pintos-Morell G, Aldámiz-Echevarría L. Tyrosinemia type 1 in Spain:
Mutational analysis, treatment and long-term outcome. Pediatr Int. 2011;53:985-9.

8. Angileri F, Bergeron A, Morrow G, Lettre F, Gray G, Hutchin $\mathrm{T}$, et al. Geographical and Ethnic Distribution of Mutations of the Fumarylacetoacetate Hydrolase Gene in Hereditary Tyrosinemia Type 1. JIMD Rep. 2015; 19:43-58.

9. Zytkovicz TH, Sahai I, Rush A, Odewale A, Johnson $D$, Fitzgerald $E$, et al. Newborn screening for hepatorenaltyrosinemia-I by tandem mass spectrometry using pooled samples: a four-year summary by the New England newborn screening program. ClinBiochem. 2013;46:681-4.

10. Fernández-Lainez C, Ibarra-González I, BelmontMartínez L, Monroy-Santoyo S, Guillén-López S, Vela-Amieva M. Tyrosinemia type 1: clinical and biochemical analysis of patients in Mexico. Ann Hepatol. 2014;13:265-72.

11. Holme E, Lindstedt S. Tyrosinemia type 1 and NTBC (2-(2-nitro-4-trifluoromethylbenzoyl)-1,3-cyclohexanedione). J Inherit Metab Dis. 1998;21:507-17.

12. Gentz J, Jagenburg R, Zetterstroem R. Tyrosinemia. J Pediatr. 1965;66:670-96.

13. Gentz J, Johansson S, Lindblad B, Lindstedt S, Zetterström R.Exertion of delta-aminolevulinic acid in hereditary tyrosinemia. ClinChimActa. 1969;23:257-63.

14. Mitchell G, Larochelle J, Lambert M, Michaud J, Grenier A, Ogier $\mathrm{H}$, et al. Neurologic crises in hereditary tyrosinemia. N Engl J Med. 1990;322:432-7.

15. Lindstedt S, Holme E, Lock EA, Hjalmarrson T, Strandvik B. Treatment of hereditary tyrosinemia type I by inhibition of 4-hydroxyphenylpyruvate dioxygenase. Lancet. 1992;340:813-17.

16. Bartlett DC, Lloyd C, McKiernan PJ, Newsome PN. Early nitisinone treatment reduces the need for liver transplantation in children with tyrosinaemia type 1 and improves post-transplant renal function. J Inherit Metab Dis. 2014;37:745-52.

17. Acosta PB, Matalon KM. Nutrition management of patients with inherited disorders of aromatic amino acid metabolism. In Acosta PB, Nutrition Management of Patients with Inherited Metabolic Disorders. Boston Mass; Jones and Bartlett Publishers, 2010:119-174.

18. Holme E, Lindstedt S. Nontransplant treatment of tyrosinemia. Clin Liver Dis. 2000;4:805-14.

19. Larochelle J, Alvarez F, Bussières JF, Chevalier I, Dallaire L, Dubois J,et al. Effect of nitisinone (NTBC) treatment on the clinical course of hepatorenaltyrosinemia in Québec. Mol Genet Metab. 2012;107:49-54. 
Ibarra González I et al. Crisis neuropática en tirosinemia

20. Schlump JU, Perot C, Ketteler K, Schiff M, Mayatepek E, Wendel $U$, et. al. Severe neurological crisis in a patient with hereditary tyrosinaemia type I afterinterruption of NTBC treatment. J Inherit Metab Dis. 2008;Suppl 2:S223-5. DOI:10.1007/s10545-008-0807-z.

21. Mungan NO, Yildizdaş D, Kör D, Horoz ÖÖ, Incecik F, Öktem M, et. al. Tyrosinemia type 1 and irreversible neurologic crisis after one-monthdiscontinuation of nitisone. Metab Brain Dis. 2016. DOI 10.1007/s11011016-9833-y.

22. Nigenda G, González-Robledo LM, Juárez-Ramírez C, Adam T. Understanding the dynamics of the Seguro Popular de Salud policy implementation in Mexico from a complex adaptive systems perspective. Implement Sci. 2016;11:68. doi:10.1186/s13012-016-0439-x. 